\title{
Anesthesia and Exsanguination Euthanasia
}

National Cancer Institute

\section{Source}

National Cancer Institute. Anesthesia and Exsanguination Euthanasia. NCI Thesaurus.

Code C90357.

A method of euthanization whereby a subject is anesthetized and the body is drained of blood. 